\title{
Extinction Phenomenon and Decay Estimate for a Quasilinear Parabolic Equation with a Nonlinear Source
}

\author{
Dengming Liu (i) and Luo Yang (it) \\ School of Mathematics and Computational Science, Hunan University of Science and Technology, Xiangtan, Hunan 411201, China \\ Correspondence should be addressed to Dengming Liu; liudengming@hnust.edu.cn
}

Received 16 January 2021; Revised 2 February 2021; Accepted 4 February 2021; Published 20 February 2021

Academic Editor: Kamyar Hosseini

Copyright @ 2021 Dengming Liu and Luo Yang. This is an open access article distributed under the Creative Commons Attribution License, which permits unrestricted use, distribution, and reproduction in any medium, provided the original work is properly cited.

By energy estimate approach and the method of upper and lower solutions, we give the conditions on the occurrence of the extinction and nonextinction behaviors of the solutions for a quasilinear parabolic equation with nonlinear source. Moreover, the decay estimates of the solutions are studied.

\section{Introduction}

The main goal of this article is to investigate the extinction behavior and decay estimate of the following parabolic initial boundary value problem

$$
\begin{cases}u_{t}=\operatorname{div}\left(u^{\alpha}|\nabla u|^{m-1} \nabla u\right)+\lambda u^{p} \int_{\Omega} u^{q} d x, & (x, t) \in \Omega \times(0,+\infty), \\ u(x, t)=0, & (x, t) \in \partial \Omega \times(0,+\infty), \\ u(x, 0)=u_{0}(x), & x \in \bar{\Omega} .\end{cases}
$$

Here, $\Omega \subset R^{N}, N \geq m+1$, is an open bounded domain with smooth boundary $\partial \Omega, m, p, q$, and $\lambda$ that are positive parameters, $0<m+\alpha<1$, and $u_{0}^{m+\alpha / m} \in L^{\infty}(\Omega) \cap W_{0}^{1, m+1}(\Omega)$ is a nonzero nonnegative function.

It is well known that this type of equation describes lots of phenomena in nature, such as heat transfer, chemical reactions, and population dynamics (one can see [1-4] for more detailed physical background). In particular, problem (1) can be used to describe the nonstationary flows in a porous medium of fluids with a power dependence of the tangential stress on the velocity of displacement under polytropic conditions. In this physical context, $u(x, t)$ is the density of the fluid, $u^{\alpha}|\nabla u|^{m-1} \nabla u$ denotes the momentum velocity, and $\lambda u^{p} \int_{\Omega} u^{q} d x$ stands for the nonlinear nonlocal source. The parameter $m$ acts as a characteristic of the medium, to be exact, the medium with $m=1$ is called Newtonian fluid, the medium with $m>1$ is called dilatant fluid, and that with 0 $<m<1$ is called pseudoplastic.

Extinction phenomenon, as one of the most remarkable properties that distinguish nonlinear parabolic problems from the linear ones, attracted extensive attentions of mathematicians in the past few decades (see [5-16] and the references therein). Especially, many authors devoted to concern with the extinction behavior of the following parabolic problem

$$
\begin{cases}u_{t}-\operatorname{div}(a(x, t, u, \nabla \varphi(u)))=f(x, t, u), & (x, t) \in \Omega \times(0,+\infty), \\ u(x, t)=0, & (x, t) \in \partial \Omega \times(0,+\infty), \\ u(x, 0)=u_{0}(x), & x \in \bar{\Omega} .\end{cases}
$$

$\mathrm{Gu}$ [17] discussed (2) with $a(x, t, u, \nabla \varphi(u))=\nabla u$ and $f(x, t, u)=-u^{p}$, and concluded that the extinction phenomenon occurs if and only if $p \in(0,1)$. Tian and $\mathrm{Mu}$ [18] dealt with problem (2) with $a(x, t, u, \nabla \varphi(u))=|\nabla u|^{p-2} \nabla u$ and $f(x$ $, t, u)=\lambda u^{p}$, and derived that $q=p-1$ is the critical extinction exponent of problem (2). The authors of $[19,20]$ generalized the results in [18] to $a(x, t, u, \nabla \varphi(u))=\left|\nabla u^{m}\right|^{p-2} \nabla u^{m}$. 
The authors of $[5,21]$ concerned with the extinction behavior of problem (2) with a $(x, t, u, \nabla \varphi(u))=\left|\nabla u^{m}\right|^{p-2} \nabla u^{m}$ and $f(x$ , $t, u)=\lambda \int_{\Omega} u^{q} d x$, and they pointed out that the effect of the nonlocal source term $\lambda \int_{\Omega} u^{q} d x$ on the extinction behavior is very different from that of the local source $\lambda u^{q}$. Recently, Zhou and Yang [22] dealt with the extinction singularity of problem (2) in the case $a(x, t, u, \nabla \varphi(u))=\nabla u^{m}$ and $f(x, t, u)$ $=\lambda u^{p} \int_{\Omega} u^{q} d x$. For some relevant works on other types of nonlinear evolution equations, the readers can refer to the references [23-28].

However, to our best knowledge, there is no literature on the study of the extinction and decay estimate of the solutions for problem (1). Motivated by those works above, we consider the extinction property of problem (1). More precisely, our purpose is to understand how the nonlinear nonlocal source affects the extinction behavior of problem (1). In other words, the aim of this article is to evaluate the competition between the diffusion term which may produce extinction phenomenon and the nonlinear nonlocal source which may prevent the occurrence of the extinction phenomenon. We want to find a critical extinction exponent and give a complete classification on the extinction and nonextinction cases of the solutions to problem (1). Meanwhile, we will deal with the decay estimates of the extinction solutions.

Since equation (1) is degenerate (or singular) at the points where $u=0$ or $\nabla u=0$, there is no classical solution in general, and hence we consider the nonnegative solution of (1) in some weak sense.

Definition 1. Let $\Sigma_{T}=\Omega \times(0, T)$, and

$$
\begin{aligned}
\mathfrak{S}= & \left\{u \in L^{2 p}\left(\Sigma_{T}\right) \cap L^{2 q}\left(\Sigma_{T}\right) \cap L^{2}\left(\Sigma_{T}\right) ; u \in C\right. \\
& \left.\cdot\left([0, T] ; L^{1}(\Omega)\right) ; \nabla u^{\frac{m+\alpha}{m}} \in L^{m+1}\left(\Sigma_{T}\right)\right\} .
\end{aligned}
$$

We say that a function $u(x, t) \in \mathbb{S}$ is a weak lower solution of problem (1) if

$$
\begin{gathered}
\int_{\Omega} u(x, T) \zeta(x, T) d x+\iint_{\Sigma_{T}}\left[u^{\alpha}|\nabla u|^{m-1} \nabla u \cdot \nabla \zeta-u \zeta_{t}\right] d x d t \\
\quad \leq \int_{\Omega} u(x, 0) \zeta(x, 0) d x+\iint_{\Sigma_{T}}\left(\lambda u^{p} \int_{\Omega} u^{q} d x\right) \zeta d x d t
\end{gathered}
$$

holds for any $T>0$ and any nonnegative test function

$\zeta \in\left\{u \in L^{2}\left(\Sigma_{T}\right) ; u \in C\left([0, T] ; L^{2}(\Omega)\right) ; u_{t} \in L^{2}\left(\Sigma_{T}\right) ; \nabla u \in L^{m+1}\left(\Sigma_{T}\right) ;\left.u\right|_{\partial \Omega}=0\right\}$.

Moreover,

$u(x, 0) \leq u_{0}(x)$ for $x \in \bar{\Omega}$, and $u(x, t) \leq 0$ for $(x, t) \in \partial \Omega \times(0, T)$

Replacing " $\leq$ " by " $\geq$ " in the inequalities (4) and (6) leads to the definition of the weak upper solution of problem (1). We say that $u$ is a weak solution of problem (1) in $\Sigma_{T}$ if it is both a weak lower solution and a weak upper solution of problem (1) in $\Sigma_{T}$.

Proposition 2. Assume that $u_{0}(x)$ is a nonzero nonnegative function satisfying $u_{0}^{m+\alpha / m} \in L^{\infty}(\Omega) \cap W_{0}^{1, m+1}(\Omega)$. Then, problem (1) has at least one local weak solution $u(x, t) \in \mathbb{S}$.

Remark 3. The proof of Proposition 2 is based on an approximation procedure and the Leray-Schauder fixed-point theorem, and it is standard and lengthy; so, we omit it here, while one can refer to the proof of Proposition 2.1 in [5] (or Proposition 2.3 in [19]) for more details. On the other hand, it is necessary to point out that the weak solution of problem (1) is unique for $p \geq 1$ and $q \geq 1$. In the non-Lipschitz case 0 $<p<1$ or $0<q<1$, the uniqueness of the weak solution seems to be unknown (See Remark 44.1 of $\$ 44.1$ in [29]).

The main results of this article are stated as follows.

Theorem 4. Assume that $0<m+\alpha<p+q$. Then, the nonnegative weak solution of problem (1) vanishes in finite time provided that the nonnegative initial datum $u_{0}(x)$ is sufficiently small. Moreover,

$$
\begin{cases}\|u\|_{\frac{2 m+\alpha}{m}} \leq\left\|u_{0}\right\|_{\frac{2 m+\alpha}{m}}\left(1-d_{4} t\right)^{\frac{1}{1-m-\alpha}}, & t \in\left[0, T_{1}\right), \\ \|u\|_{\frac{2 m+\alpha}{m}} \equiv 0, & t \in\left[T_{1},+\infty\right),\end{cases}
$$

for $\quad m(N-m-1 / N m+m+1-1) \leq \alpha<1, \quad$ and $\quad\{\| u$ $\left\|_{N(1-m-\alpha) / m+1} \leq\right\| u_{0} \|_{N(1-m-\alpha) / m+1}\left(1-d_{8} t\right)^{1 / 1-(m+\alpha)}, t \in\left[0, T_{2}\right)$, $\|u\|_{N(1-m-\alpha) / m+1} \equiv 0, t \in\left[T_{2},+\infty\right)$,

for $-m<\alpha<m(N-m-1 / N m+m+1-1)$, where $T_{1}=$ $d_{4}^{-1}, T_{2}=d_{8}^{-1}, d_{4}$, and $d_{8}$ are positive constants, given in Section 2.

Theorem 5. Assume that $0<p+q<m+\alpha<1$ and $\lambda$ are sufficiently large. Then, for any nonnegative initial datum $u_{0}(x)$, problem (1) admits at least one nonextinction weak solution.

Theorem 6. Assume that $0<m+\alpha=p+q<1$.

(1) The nonnegative weak solution of problem (1) vanishes in finite time provided that $\lambda$ is sufficiently small. Moreover,

$$
\begin{cases}\|u\|_{\frac{2 m+\alpha}{m}} \leq\left\|u_{0}\right\|_{\frac{2 m+\alpha}{m}}\left(1-d_{14} t\right)^{\frac{1}{1-m-\alpha}}, & t \in\left[0, T_{3}\right), \\ \|u\|_{\frac{2 m+\alpha}{m}} \equiv 0, & t \in\left[T_{3},+\infty\right)\end{cases}
$$

$$
\text { for } m(N-m-1 / N m+m+1-1) \leq \alpha<1 \text {, and }\{\| u
$$
$\left\|_{N(1-m-\alpha) / m+1} \leq\right\| u_{0} \|_{N(1-m-\alpha) / m+1}\left(1-d_{15} t\right)^{1 / 1-m-\alpha}, t \in\left[0, T_{4}\right)$, $\|u\|_{N(1-m-\alpha) / m+1} \equiv 0, t \in\left[T_{4},+\infty\right)$, 
for $-m<\alpha<m(N-m-1 / N m+m+1-1)$, where $T_{3}=$ $d_{14}^{-1}, T_{4}=d_{15}^{-1}, d_{14}$, and $d_{15}$ are positive constants, given in Section 2.

(2) Problem (1) admits at least one non-extinction weak solution for any nonnegative initial datum $u_{0}(x)$ provided that $\lambda$ is sufficiently large

\section{Proofs of the Main Results}

In this section, based on energy estimates approach and the method of upper and lower solutions, we will give the proofs of our main results.

Proof of Theorem 4. Multiplying equation (1) by $u^{s}$ and integrating over $\Omega$, one has

$$
\begin{aligned}
& \frac{1}{s+1} \frac{d}{d t} \int_{\Omega} u^{s+1} d x+s\left(\frac{m+1}{m+\alpha+s}\right)^{m+1} \int_{\Omega}\left|\nabla u^{\frac{m+\alpha+s}{m+1}}\right|^{m+1} d x \\
& \quad=\lambda \int_{\Omega} u^{p+s} d x \int_{\Omega} u^{q} d x,
\end{aligned}
$$

where

$s= \begin{cases}\frac{m+\alpha}{m}, & \text { if } m\left(\frac{N-m-1}{N m+m+1}-1\right) \leq \alpha<1, \\ \frac{N(1-m-\alpha)-m-1}{m+1}, & \text { if }-m<\alpha<m\left(\frac{N-m-1}{N m+m+1}-1\right) .\end{cases}$

We now divide the proof into two cases according to the different values of $p+q$.

Case 1. $m+\alpha<p+q \leq 1$. For $m(N-m-1 / N m+m+1-1)$ $\leq \alpha<1$. It follows from Hölder inequality and (9) that

$$
\begin{aligned}
& \frac{m}{2 m+\alpha} \frac{d}{d t} \int_{\Omega} u^{\frac{2 m+\alpha}{m}} d x+\left(\frac{m}{m+\alpha}\right)^{m} \int_{\Omega}\left|\nabla u^{\frac{m+\alpha}{m}}\right|^{m+1} d x \\
& \leq \lambda|\Omega|^{2-\frac{m(p+q)+m+\alpha}{2 m+\alpha}}\left(\int_{\Omega} u^{\frac{2 m+\alpha}{m}} d x\right)^{\frac{m(p+q)+m+\alpha}{2 m+\alpha}} .
\end{aligned}
$$

Using Hölder inequality and Sobolev embedding theorem, one has

$$
\begin{aligned}
\int_{\Omega} u^{\frac{2 m+\alpha}{m}} d x & \leq|\Omega|^{1-\frac{(2 m+\alpha)(N-m-1)}{N(m+1)(m+\alpha)}}\left(\int_{\Omega} u^{\frac{m+\alpha}{m} \cdot \frac{N(m+1)}{N-m-1}} d x\right)^{\frac{2 m+\alpha}{m+\alpha} \cdot \frac{N-m-1}{N(m+1)}} \\
& \leq \kappa_{1}|\Omega|^{1-\frac{(2 m+\alpha)(N-m-1)}{N(m+1)(m+\alpha)}}\left(\int_{\Omega}\left|\nabla u^{\frac{m+\alpha}{m}}\right|^{m+1} d x\right)^{\frac{2 m+\alpha}{(m+1)(m+\alpha)}}
\end{aligned}
$$

which is equivalent to

$$
\kappa_{1}^{-\frac{(m+1)(m+\alpha)}{2 m+\alpha}}|\Omega|^{1-\frac{m+1}{N}-\frac{(m+1)(m+\alpha)}{2 m+\alpha}}\left(\int_{\Omega} \mathcal{U}^{\frac{2 m+\alpha}{m}} d x\right)^{\frac{(m+1)(m+\alpha)}{2 m+\alpha}} \leq \int_{\Omega}\left|\nabla u^{\frac{m+\alpha}{m}}\right|^{m+1} d x
$$

where $\kappa_{1}=\kappa_{1}(\alpha, m, N)$ is the embedding constant. Inserting (13) into (11) yields

$\frac{d}{d t} \int_{\Omega} u^{\frac{2 m+\alpha}{m}} d x+d_{1}\left(\int_{\Omega} u^{\frac{2 m+\alpha}{m}} d x\right)^{\frac{(m+1)(m+\alpha)}{2 m+\alpha}} \leq d_{2}\left(\int_{\Omega} u^{\frac{2 m+\alpha}{m}} d x\right)^{\frac{m(p+q)+m+\alpha}{2 m+\alpha}}$,

where

$$
\begin{aligned}
& d_{1}=(2 m+\alpha) m^{m-1}(m+\alpha)^{-m} \kappa_{1}^{-\frac{(m+1)(m+\alpha)}{2 m+\alpha}}|\Omega|^{1-\frac{m+1}{N}-\frac{(m+1)(m+\alpha)}{2 m+\alpha}}, \\
& d_{2}=\lambda(2 m+\alpha) m^{-1}|\Omega|^{2-\frac{m(p+q)+m+\alpha}{2 m+\alpha}} .
\end{aligned}
$$

Now, if $u_{0}(x)$ is sufficiently small satisfying

$$
d_{3}=d_{1}-d_{2}\left(\int_{\Omega} u_{0}^{\frac{2 m+\alpha}{m}} d x\right)^{\frac{m(p+q-m-\alpha)}{2 m+\alpha}}>0,
$$

then (14) leads to

$$
\frac{d}{d t} \int_{\Omega} u^{\frac{2 m+\alpha}{m}} d x+d_{3}\left(\int_{\Omega} u^{\frac{2 m+\alpha}{m}} d x\right)^{\frac{(m+1)(m+\alpha)}{2 m+\alpha}} \leq 0
$$

By integration, one can deduce that

$$
\|u\|_{\frac{2 m+\alpha}{m}} \leq\left\|u_{0}\right\|_{\frac{2 m+\alpha}{m}}\left(1-d_{4} t\right)_{+}^{\frac{1}{1-m-\alpha}}
$$

which tells us that $u(x, t)$ vanishes in finite time $T_{1}=d_{4}^{-1}$, where

$$
d_{4}=m d_{3}(1-m-\alpha)(2 m+\alpha)^{-1}\left\|u_{0}\right\|_{\frac{2 m+\alpha}{m}}^{m+\alpha-1} .
$$

For $-m<\alpha<m(N-m-1 / N m+m+1-1)$. By Sobolev embedding theorem, one obtains

$$
\left(\int_{\Omega} u^{s+1} d x\right)^{\frac{m+\alpha+s}{(m+1)(s+1)}}=\left(\int_{\Omega} u^{\frac{N(\alpha+m+s)}{N-m-1}} d x\right)^{\frac{N-m-1}{N(m+1)}} \leq \kappa_{2}\left(\int_{\Omega}\left|\nabla u^{\frac{\alpha+m+s}{m+1}}\right|^{m+1} d x\right)^{\frac{1}{m+1}} .
$$

Here, $\kappa_{2}=\kappa_{2}(\alpha, m, N)$ is the embedding constant. Combining (9) and (20), and in view of Hölder inequality, one arrives at

$$
\frac{d}{d t} \int_{\Omega} u^{s+1} d x+d_{5}\left(\int_{\Omega} u^{s+1} d x\right)^{\frac{m+\alpha+s}{s+1}} \leq d_{6}\left(\int_{\Omega} u^{s+1} d x\right)^{\frac{p+q+s}{s+1}},
$$


where

$$
\begin{aligned}
& d_{5}=s(s+1)\left[(m+1)\left(\kappa_{2}(m+\alpha+s)\right)^{-1}\right]^{m+1}, \\
& d_{6}=\lambda(s+1)|\Omega|^{2-\frac{p+q+s}{s+1}} .
\end{aligned}
$$

Next, choosing $u_{0}(x)$ sufficiently small such that

$$
d_{7}=d_{5}-d_{6}\left(\int_{\Omega} u_{0}^{s+1} d x\right)^{\frac{p+q-m-\alpha}{s+1}}>0,
$$

then from (21), one has

$$
\frac{d}{d t} \int_{\Omega} u^{s+1} d x+d_{7}\left(\int_{\Omega} u^{s+1} d x\right)^{\frac{m+\alpha+s}{s+1}} \leq 0
$$

Integrating (24) from 0 to $t$ gives us that

$$
\|u\|_{\frac{N(1-m-\alpha)}{m+1}} \leq\left\|u_{0}\right\|_{\frac{N(1-m-\alpha)}{m+1}}\left(1-d_{8} t\right)_{+}^{\frac{1}{1-m-\alpha}},
$$

which means that $u(x, t)$ vanishes in finite time $T_{2}=d_{8}^{-1}$, where

$$
d_{8}=d_{7}(m+1)(1-m-\alpha)[N(1-m-\alpha)]^{-1} \| u_{0} \frac{\|_{N(1-m-\alpha)}^{m+\alpha}}{m+1} .
$$

Case 2. $m+\alpha<1<p+q$. If $p<1$ or $q<s+1$, then the proof is the same as that in Case 1 . We only need to focus our attention on the subcase $p \geq 1$ and $q \geq s+1$. Let $\tilde{\Omega}$ be a bounded domain in $R^{N}$ satisfying $\Omega \subset \subset \tilde{\Omega}$. Denote $\tilde{\lambda}_{1}$ be the first eigenvalue and $\tilde{\Psi}(x)$ be the corresponding eigenfunction of problem (One can see Lemma 2.3 of [18] for more details on the properties of the first eigenvalue and the corresponding eigenfunction of (27).)

$$
\begin{cases}-\operatorname{div}\left(\mathscr{U}^{\alpha}|\nabla \mathscr{U}|^{m-1} \nabla \mathscr{U}\right)=\lambda \mathcal{U}^{\alpha+1}|\mathscr{U}|^{m-1}, & x \in \tilde{\Omega}, \\ \mathscr{U}(x)=0, & x \in \partial \tilde{\Omega} .\end{cases}
$$

We assume that $\max _{x \in \tilde{\Omega}} \tilde{\Psi}(x)=1$. Put

$\mathrm{U}_{1}(x, t)=\mu \tilde{\Psi}(x)$ with $\mu \in\left(\max _{x \in \bar{\Omega}} \frac{u_{0}(x)}{\tilde{\Psi}(x)},\left(\min _{x \in \bar{\Omega}} \frac{\tilde{\lambda}_{1} \tilde{\Psi}^{m+\alpha}(x)}{\lambda|\Omega|}\right)^{\frac{1}{p+q-m-\alpha}}\right)$.

Then, it is not difficult to show that $\mathrm{U}_{1}(x, t)$ is an upper solution of problem (1). Therefore, one has $u(x, t) \leq \mu \tilde{\Psi}(x)$ $\leq \mu$ and

$$
\lambda \int_{\Omega} u^{p+s} d x \int_{\Omega} u^{q} d x \leq \lambda|\Omega| \mu^{p+q-1} \int_{\Omega} u^{s+1} d x
$$

It follows from (9) and (29) that

$$
\begin{aligned}
& \frac{1}{s+1} \frac{d}{d t} \int_{\Omega} u^{s+1} d x+s\left(\frac{m+1}{m+\alpha+s}\right)^{m+1} \int_{\Omega}\left|\nabla u^{\frac{m+\alpha+s}{m+1}}\right|^{m+1} d x \\
& \quad \leq \lambda|\Omega| \mu^{p+q-1} \int_{\Omega} u^{s+1} d x .
\end{aligned}
$$

For $m(N-m-1 / N m+m+1-1) \leq \alpha<1$. It follows from (13) and (30) that

$$
\frac{d}{d t} \int_{\Omega} u^{\frac{2 m+\alpha}{m}} d x+d_{1}\left(\int_{\Omega} u^{\frac{2 m+\alpha}{m}} d x\right)^{\frac{(m+1)(m+\alpha)}{2 m+\alpha}} \leq d_{9} \int_{\Omega} u^{\frac{2 m+\alpha}{m}} d x,
$$

where

$$
d_{9}=\lambda|\Omega|(2 m+\alpha) m^{-1} \mu^{p+q-1} .
$$

Now, selecting $u_{0}(x)$ sufficiently small satisfying

$$
d_{10}=d_{1}-d_{9}\left(\int_{\Omega} u_{0}^{\frac{2 m+\alpha}{m}} d x\right)^{\frac{m(1-m-\alpha)}{2 m+\alpha}}>0,
$$

then (31) tells us that

$$
\frac{d}{d t} \int_{\Omega} u^{\frac{2 m+\alpha}{m}} d x+d_{10}\left(\int_{\Omega} u^{\frac{2 m+\alpha}{m}} d x\right)^{\frac{(m+1)(m+\alpha)}{2 m+\alpha}} \leq 0 .
$$

A simple integration of $(34)$ over $(0, t)$ gives

$$
\|u\|_{\frac{2 m+\alpha}{m}} \leq\left\|u_{0}\right\|_{\frac{2 m+\alpha}{m}}\left(1-d_{4} t\right)_{+}^{\frac{1}{1-m-\alpha}}
$$

which means that $u(x, t)$ vanishes in finite time, where

$$
d_{4}=m d_{10}(1-m-\alpha)(2 m+\alpha)^{-1}\left\|u_{0}\right\|_{\frac{2 m+\alpha}{m}}^{m+\alpha-1} .
$$

For $-m<\alpha<m(N-m-1 / N m+m+1-1)$. Recalling (20) and (30), one obtains

$$
\frac{d}{d t} \int_{\Omega} u^{s+1} d x+d_{5}\left(\int_{\Omega} u^{s+1} d x\right)^{\frac{m+\alpha+s}{s+1}} \leq d_{11} \int_{\Omega} u^{s+1} d x
$$

where

$$
d_{11}=\lambda(s+1)|\Omega| \mu^{p+q-1} .
$$

Next, if $u_{0}(x)$ is sufficiently small such that

$$
d_{12}=d_{5}-d_{11}\left(\int_{\Omega} u_{0}^{s+1} d x\right)^{\frac{1-m-\alpha}{s+1}}>0
$$


then from (37), one arrives at

$$
\frac{d}{d t} \int_{\Omega} u^{s+1} d x+d_{12}\left(\int_{\Omega} u^{s+1} \mathrm{~d} x\right)^{\frac{m+\alpha+s}{s+1}} \leq 0
$$

Integrating (40), one can claim that

$$
\|u\|_{\frac{N(1-m-\alpha)}{m+1}} \leq\left\|u_{0}\right\|_{\frac{N(1-m-\alpha)}{m+1}}\left(1-d_{8} t\right)_{+}^{\frac{1}{1-m-\alpha}}
$$

which tells us that $u(x, t)$ vanishes in finite time, where

$$
d_{8}=d_{12}(m+1) N^{-1}\left\|u_{0}\right\|_{\frac{N(1-m-\alpha)}{m+1}}^{m+\alpha-1} .
$$

The proof of Theorem 4 is complete.

Proof of Theorem 5. Let $\lambda_{1}$ be the first eigenvalue and $\Psi(x)$ be the corresponding eigenfunction of the following problem

$$
\begin{cases}-\operatorname{div}\left(\mathscr{U}^{\alpha}|\nabla \mathscr{U}|^{m-1} \nabla \mathcal{U}\right)=\lambda \mathcal{U}^{\alpha+1}|\mathcal{U}|^{m-1}, & x \in \Omega, \\ \mathscr{U}(x)=0, & x \in \partial \Omega .\end{cases}
$$

In what follows, we assume that $\Psi(x)>0$ and $\max _{x \in \Omega} \Psi(x)$ $=1$. Define $f(t)=\left(1-e^{-c t}\right)^{1 / 1-p-q}$, where $c \in(0,(1-p-q)$ $\left.\left(\lambda\|\Psi\|_{q}^{q}-\lambda_{1}\right)\right)$. Then, it is easy to check that

$$
f(0)=0 \text {, and } f(t) \in(0,1) \text { for } t>0 \text {. }
$$

In addition, one has

$$
f^{\prime}(t)+\lambda_{1} f^{m+\alpha}(t)-\lambda\|\Psi\|_{q}^{q} f^{p+q}(t) \leq 0 .
$$

Define $\mathrm{U}_{2}(x, t)=f(t) \Psi(x)$. Then, one can verify that

$$
\begin{aligned}
\mathrm{U}_{2 t}- & \operatorname{div}\left(\mathrm{U}_{2}^{\alpha}\left|\nabla \mathrm{U}_{2}\right|^{m-1} \nabla \mathrm{U}_{2}\right)-\lambda \mathrm{U}_{2}^{p} \int_{\Omega} \mathrm{U}_{2}^{q} d x \\
= & f^{\prime}(t) \Psi(x)+\lambda_{1} f^{m+\alpha}(t) \Psi^{m+\alpha}(x)-\lambda\|\Psi\|_{q}^{q} f^{p+q}(t) \Psi^{p}(x) \\
& <\left(f^{\prime}(t)+\lambda_{1} f^{m+\alpha}(t)-\lambda\|\Psi\|_{q}^{q} f^{p+q}(t)\right) \Psi^{p}(x) \leq 0,
\end{aligned}
$$

which implies that $\mathrm{U}_{2}(x, t)$ is a strict weak lower solution of problem (1) if $\lambda>\lambda_{1}\|\Psi\|_{q}^{-q}$.

Now, consider the following problem

$$
\begin{cases}u_{t}=\operatorname{div}\left(u^{\alpha}|\nabla u|^{m-1} \nabla u\right)+\lambda\left(u_{+}+1\right)^{p} \int_{\Omega}\left(u_{+}+1\right)^{q} d x, & (x, t) \in \Omega \times(0, \infty), \\ u(x, t)=0, & (x, t) \in \partial \Omega \times(0, \infty), \\ u(x, 0)=u_{0}(x) \geq 0, & x \in \bar{\Omega} .\end{cases}
$$

Using Leray-Schauder fixed-point theorem, we can prove that problem (47) admits at least one weak solution $\mathrm{U}_{3}(x, t)$, and we know that $\mathrm{U}_{3}(x, t) \geq 0$ by the weak maximum princi- ple. In addition, the weak solution $\mathrm{U}_{3}(x, t)$ is also a weak upper solution of problem (1).

Up to now, we have constructed a pair of weak upper and lower solutions $\mathrm{U}_{3}(x, t), \mathrm{U}_{2}(x, t)$. If $\mathrm{U}_{2}(x, t) \leq \mathrm{U}_{3}(x, t)$, then problem (1) admits a weak solution $\tilde{u}$ satisfying $U_{2} \leq \tilde{u} \leq$ $\mathrm{U}_{3}$. By the definitions of $\mathrm{U}_{2}$ and $\mathrm{U}_{3}$, one has

$$
\begin{aligned}
\int_{\Omega}\left(\mathrm{U}_{2}(x, t)-\mathrm{U}_{3}(x, t)\right) \zeta(x, t) d x-\int_{\Omega} \\
\quad \cdot\left(\mathrm{U}_{2}(x, 0)-\mathrm{U}_{3}(x, 0)\right) \zeta(x, 0) d x \\
\quad+\iint_{\Sigma_{t}}\left(\mathrm{U}_{2}^{\alpha}\left|\nabla \mathrm{U}_{2}\right|^{m-1} \nabla \mathrm{U}_{2}-\mathrm{U}_{3}^{\alpha}\left|\nabla \mathrm{U}_{3}\right|^{m-1} \nabla \mathrm{U}_{3}\right) \cdot \nabla \zeta d x d \tau \\
\quad-\iint_{\Sigma_{t}}\left(\mathrm{U}_{2}-\mathrm{U}_{3}\right) \zeta_{\tau} d x d \tau \\
\leq \lambda \iint_{\Sigma_{t}}\left(\mathrm{U}_{2}^{p} \int_{\Omega} \mathrm{U}_{2}^{q} d x-\left(\mathrm{U}_{3+}+1\right)^{p} \int_{\Omega}\left(\mathrm{U}_{3+}+1\right)^{q} d x\right) \zeta d x d \tau \\
=\lambda \iint_{\Sigma_{t}}\left[\mathrm{U}_{2}^{p} \int_{\Omega}\left(\mathrm{U}_{2}^{q}-\left(\mathrm{U}_{3+}+1\right)^{q}\right) d x+\left(\mathrm{U}_{2}^{p}-\left(\mathrm{U}_{3+}+1\right)^{p}\right)\right. \\
\left.\quad \cdot \int_{\Omega}\left(\mathrm{U}_{3+}+1\right)^{q} d x\right] \zeta d x d \tau .
\end{aligned}
$$

Take $\zeta(x, t)=H_{\varepsilon}\left(\mathrm{U}_{2}^{m+\alpha / m}-\mathrm{U}_{3}^{m+\alpha / m}\right)$, where $H_{\varepsilon}(r)$ is a monotone increasing smooth approximation of the following function

$$
H(r)= \begin{cases}1, & r>0 \\ 0, & \text { otherwise }\end{cases}
$$

It is easy to check that $H_{\varepsilon}^{\prime}(r) \rightarrow \delta(r)$ as $\varepsilon \rightarrow 0$. Letting $\varepsilon$ $\rightarrow 0$, it follows from (48) that

$$
\begin{aligned}
\int_{\Omega}\left(\mathrm{U}_{2}-\mathrm{U}_{3}\right)_{+} d x \leq & \lambda \iint_{\Sigma_{t}}\left[\mathrm{U}_{2}^{p} \int_{\Omega}\left(\mathrm{U}_{2}^{q}-\left(\mathrm{U}_{3+}+1\right)^{q}\right) d x\right] H \\
& \cdot\left(\mathrm{U}_{2}^{\frac{m+\alpha}{m}}-\mathrm{U}_{3}^{\frac{m+\alpha}{m}}\right) d x d \tau+\lambda \iint_{\Sigma_{t}} \\
& \cdot\left[\left(\mathrm{U}_{2}^{p}-\left(\mathrm{U}_{3+}+1\right)^{p}\right) \int_{\Omega}\left(\mathrm{U}_{3+}+1\right)^{q} d x\right] H \\
& \cdot\left(\mathrm{U}_{2}^{\frac{m+\alpha}{m}}-\mathrm{U}_{3}^{\frac{m+\alpha}{m}}\right) d x d \tau \\
\leq & d_{13} \iint_{\Sigma_{t}}\left(\mathrm{U}_{2}-\mathrm{U}_{3}\right)_{+} d x d \tau,
\end{aligned}
$$

where $d_{13}$ is a positive constant. Using Gronwall's inequality, one can conclude that $\mathrm{U}_{2}(x, t) \leq \mathrm{U}_{3}(x, t)$, a.e., in $\Omega \times(0, \infty)$. Furthermore, since $\mathrm{U}_{2}$ does not vanish, neither does $\tilde{u}$. The proof of Theorem 5 is complete.

Proof of Theorem 6.

(1) For $m(N-m-1 / N m+m+1-1) \leq \alpha<1$. It follows from (14) that 


$$
\frac{d}{d t} \int_{\Omega} u^{\frac{2 m+\alpha}{m}} d x \leq\left(d_{2}-d_{1}\right)\left(\int_{\Omega} u^{\frac{2 m+\alpha}{m}} d x\right)^{\frac{(m+1)(m+\alpha)}{2 m+\alpha}}
$$

If $\lambda$ is sufficiently small such that $d_{1}-d_{2} \geq 0$, then above inequality tells us that

$$
\|u\|_{\frac{2 m+\alpha}{m}} \leq\left\|u_{0}\right\|_{\frac{2 m+\alpha}{m}}\left(1-d_{14} t\right)_{+}^{\frac{1}{1-m-\alpha}},
$$

which means that $u(x, t)$ vanishes in finite time $T_{3}=d_{14}^{-1}$, where

$$
d_{14}=m\left(d_{1}-d_{2}\right)(1-m-\alpha)(2 m+\alpha)^{-1}\left\|u_{0}\right\|_{\frac{2 m+\alpha}{m}}^{m+\alpha-1} .
$$

For $-m<\alpha<m(N-m-1 / N m+m+1-1)$. It follows from (21) that

$$
\frac{d}{d t} \int_{\Omega} u^{\frac{N(1-m-\alpha)}{m+1}} d x \leq\left(d_{6}-d_{5}\right)\left(\int_{\Omega} u^{\frac{N(1-m-\alpha)}{m+1}} d x\right)^{\frac{N-m-1}{N}} .
$$

If $\lambda$ is sufficiently small such that $d_{5}-d_{6} \geq 0$, then (54) leads to

$$
\|u\|_{\frac{N(1-m-\alpha)}{m+1}} \leq\left\|u_{0}\right\|_{\frac{N(1-m-\alpha)}{m+1}}\left(1-d_{15} t\right)_{+}^{\frac{1}{1-m-\alpha}}
$$
where

which implies that $u(x, t)$ vanishes in finite time $T_{4}=d_{15}^{-1}$,

$$
d_{15}=(m+1)\left(d_{5}-d_{6}\right) N^{-1}\left\|u_{0}\right\|_{\frac{2 m+\alpha}{m}}^{m+\alpha-1} .
$$

(2) Let

$$
\mathrm{U}_{4}(x, t)=\left[(1-p-q)\left(\lambda\|\Psi\|_{q}^{q}-\lambda_{1}\right) t\right]^{\frac{1}{1-p-q}} \Psi(x) .
$$

One can easily prove that $\mathrm{U}_{4}(x, t)$ is a weak nonextinction lower solution of problem (1) if $\lambda>\lambda_{1}\|\Psi\|_{q}^{-q}$. On the other hand, let $\mathrm{U}_{5}(x, t)$ be a weak solution of problem (47) with $p+q=m+\alpha$; then, $\mathrm{U}_{5}(x, t)$ is a weak upper solution of problem (1). Similar to the process of proof of Theorem 5 , one can claim that problem (1) has at least one nonextinction weak solution $\tilde{u}$. The proof of Theorem 6 is complete.

\section{Conclusion}

In the present article, we mainly focus on the extinction phenomenon and the decay estimates of the solution to a quasilinear parabolic equation with a coupled nonlinear source. By analyzing the competition between the coupled nonlinear source term and the fast diffusion term, along with energy estimates approach and the method of upper and lower solutions, we show that $p+q=m+\alpha$ is the critical extinction exponent of the solutions. That is, if $m+\alpha<p+q$, then for sufficiently small initial datum, the solution possesses extinc- tion property, while if $p+q<m+\alpha$, then for any nonnegative initial datum, problem (1) admits at least one nonextinction solution provided that $\lambda$ is sufficiently large. In the critical case $p+q=m+\alpha$, whether the solution vanishes or not depends on the size of the parameter $\lambda$.

Our next work is to study the numerical extinction phenomenon of the parabolic problems like (1). We hope to give some numerical examples for our theoretical researches in the near future.

\section{Data Availability}

The data used to support the findings of this study are included within the article.

\section{Conflicts of Interest}

Conflict of interest statement is included without existing competing interests.

\section{Acknowledgments}

The first author is sincerely grateful to professor Chunlai $\mathrm{Mu}$ of Chongqing University for his encouragements and suggestions. The authors would like to thank the editor and anonymous referee for their careful reading and important comments and suggestions. This research is supported by the Natural Science Foundation of Hunan Province (Grant No. 2019JJ50160).

\section{References}

[1] A. S. Kalashnikov, "Some problems of the qualitative theory of second-order nonlinear degenerate parabolic equations," Uspekhi Matematicheskikh Nauk, vol. 42, no. 2, pp. 135-176, 1987.

[2] J. L. Vazquez, The Porous Medium Equations: Mathematical Theory, Oxford University Press, London, 2007.

[3] Z. Wu, J. Yin, H. Li, and J. Zhao, Nonlinear Diffusion Equations, World Scientific Publishing Co., Inc., River Edge, NJ, USA, 2001.

[4] D. M. Liu and C. L. Mu, "Cauchy problem for a doubly degenerate parabolic equation with inhomogeneous source and measure data," Differential and Intergral Equations, vol. 27, no. 11/12, pp. 1001-1012, 2014.

[5] Y. Z. Han and W. J. Gao, "Extinction and non-extinction for a polytropic filtration equation with a nonlocal source," Applicable Analysis, vol. 92, no. 3, pp. 636-650, 2013.

[6] R. M. P. Almeida, S. N. Antotesv, and J. C. M. Duque, "On a nonlocal degenerate parabolic problem," Nonlinear Analysis: Real World Applications, vol. 27, pp. 146-157, 2016.

[7] Y. J. Chen, J. Wang, and H. X. Zhang, "Extinction for a couple of fast diffusion systems with nonlinear sources," Nonlinear Analysis: Real World Applications, vol. 14, no. 4, pp. 19311937, 2013.

[8] Z. B. Fang and X. H. Xu, "Extinction behavior of solutions for the p-Laplacian equations with nonlocal sources," Nonlinear Analysis: Real World Applications, vol. 13, no. 4, pp. 17801789, 2012.

[9] D. M. Liu and C. L. Mu, "Critical extinction exponent for a doubly degenerate non-divergent parabolic equation with a 
gradient source," Applicable Analysis, vol. 97, no. 12, pp. 2132$2141,2018$.

[10] D. M. Liu and C. L. Mu, "Extinction for a quasilinear parabolic equation with a nonlinear gradient source," Taiwanese Journal of Mathematics, vol. 18, no. 5, pp. 1329-1343, 2014.

[11] D. Liu and C. Mu, "Extinction for a quasilinear parabolic equation with a nonlinear gradient source and absorption," Journal of Applied Analysis \& Computation, vol. 5, no. 1, pp. 114-137, 2015.

[12] W. Liu, "Extinction properties of solutions for a class of fast diffusive p-Laplacian equations," Nonlinear Analysis: Theory, Methods \& Applications, vol. 74, no. 13, pp. 4520-4532, 2011.

[13] W. J. Liu and B. Wu, "A note on extinction for fast diffusivepLaplacian with sources," Mathematicsl Methods in the Applied Sciences, vol. 31, no. 12, pp. 1383-1386, 2008.

[14] Y. F. Wang and J. X. Yin, "Critical extinction exponents for a polytropic filtration equation with absorption and source," Mathematicsl Methods in the Applied Sciences, vol. 36, no. 12, pp. 1591-1597, 2013.

[15] J. X. Yin and C. H. Jin, "Critical extinction and blow-up exponents for fast diffusivep-Laplacian with sources," Mathematicsl Methods in the Applied Sciences, vol. 30, no. 10, pp. 1147-1167, 2007.

[16] Y. Hongjun, L. Songzhe, G. Wenjie, X. Xiaojing, and C. Chunling, "Extinction and positivity for the evolution p-Laplacian equation in $R^{n}$," Nonlinear Analysis: Theory, Methods \& Applications, vol. 60, no. 6, pp. 1085-1091, 2005.

[17] Y. G. Gu, "Necessary and sufficient conditions of extinction of solution on parabolic equations," Acta Mathematica Sinica, vol. 37, no. 1, pp. 73-79, 1994, (In Chinese).

[18] Y. Tian and C. L. Mu, "Extinction and non-extinction for a pLaplacian equation with nonlinear source," Nonlinear Analysis, vol. 69, no. 8, pp. 2422-2431, 2008.

[19] C. H. Jin, J. X. Yin, and Y. Y. Ke, "Critical extinction and blowup exponents for fast diffusive polytropic filtration equation with sources," Proceedings of the Edinburgh Mathematical Society, vol. 52, no. 2, pp. 419-444, 2009.

[20] J. Zhou and C. L. Mu, "Critical blow-up and extinction exponents for non-Newton polytropic filtration equation with source," Bulletin of the Korean Mathematical Society, vol. 46, no. 6, pp. 1159-1173, 2009.

[21] Y. Z. Han and W. J. Gao, "Extinction for a fast diffusion equation with a nonlinear nonlocal source," Archiv der Mathematik, vol. 97, no. 4, pp. 353-363, 2011.

[22] S. Zhou and Z. D. Yang, "Extinction and non-extinction behavior of solutions for a class of reaction-diffusion equations with a nonlinear source," Acta Mathematica Scientia, vol. 36A, no. 3, pp. 531-542, 2016, (In Chinese).

[23] S. Boulaaras, "Some new properties of asynchronous algorithms of theta scheme combined with finite elements methods for an evolutionary implicit 2-sided obstacle problem," Mathematical Methods in the Applied Sciences, vol. 40, no. 18, pp. 7231-7239, 2017.

[24] A. Choucha, S. Boulaaras, D. Ouchenane, and S. Beloul, "General decay of nonlinear viscoelastic Kirchhoff equation with Balakrishnan-Taylor damping, logarithmic nonlinearity and distributed delay terms," Mathematical Methods in the Applied Sciences, 2020.

[25] A. Choucha, S. Boulaaras, and D. Ouchenane, "Exponential decay of solutions for a viscoelastic coupled lame system with logarithmic source and distributed delay terms," Mathematical Methods in the Applied Sciences, 2020.

[26] S. Boulaaras and N. Doudi, "Global existence and exponential stability of coupled Lamé system with distributed delay and source term without memory term," Boundary Value Problems, vol. 2020, no. 1, 2020.

[27] N. Doudi and S. Boulaaras, "Global existence combined with general decay of solutions for coupled Kirchhoff system with a distributed delay term," Revista de la Real Academia de Ciencias Exactas, Físicas y Naturales. Serie A. Matemáticas, vol. 114, no. 4, 2020.

[28] D. M. Liu, C. L. Mu, and I. Ahmed, "Blow-up for a semilinear parabolic equation with nonlinear memory and nonlocal nonlinear boundary," Taiwanese Journal of Mathematics, vol. 17, no. 4, pp. 1353-1370, 2013.

[29] P. Quittner and P. Souplet, Superlinear Parabolic Problems: Blow-Up, Global Existence and Steady States, Birkhäuser Verlag AG, Basel, 2007. 